\title{
Processed meat intake in relation to semen quality indicators among men attending a fertility clinic
}

\author{
Anna Danielewicz, Katarzyna Przybyłowicz and Jakub Morze \\ Department of Human Nutrition, University of Warmia and Mazury, Olsztyn, Poland
}

\section{Abstract}

It is estimated that in Central and Eastern Europe 8-12\% of men are infertile, and in the case of $20 \%$ of infertile couples in the region, indicates a $56 \%$ part of the malefactor in the occurrence of infertility. Numerous studies have shown that a healthy diet with a high intake of fish and seafood, poultry, vegetables, fruits, legumes, through the high content of antioxidants are associated with better parameters of semen quality and reduced risk of asthenozoospermia. Men's diets may affect spermatogenesis as reflected in semen quality indicators, but the literature on the relation between meat intake and semen quality is limited. Our objective was to prospectively examine the relation between meats including red, poultry, proceesed and fish and indicators of semen quality. Men in subfertile couples presenting for evaluation at the Olsztyn Hospital Fertility Center were invited to participate in an ongoing study of environmental factors and fertility. This cross-sectional study was carried out in 208 men aged 18-55 years from Northern-Eastern Poland. Semen samples were collected at the clinic and analyses were performed by an experienced technician. Semen microscopic measurements were determined with the use of computer-aided semen analysis (CASA) and evaluated according to the World Health Organization (WHO) guidelines. A total of men completed a validated food-frequency questionnaire and subsequently provided semen samples. We used linear mixed regression models to examine the relation between meat intake and semen quality indicators (total sperm count, sperm concentration, progressive motility, morphology, and semen volume) while adjusting for potential confounders. After adjustment for age, BMI, physical activity, residence place and education, one serving increase in intake of processed meat was associated with a 56\% higher risk of abnormal progressive sperm motility (OR =1.56 95\%CI:1.09-2.21). Consuming processed meat may have a negative impact on sperm counts and morphology. Potentially, these products may be a significant source of saturated and trans fatty acids, xenobiotics, mainly xenoestrogen, and steroids. Is need to clarify the potential effect of meat products, especially processed meat, on male reproductive potential in RCTs or longitudinal studies. Future findings may be useful to establish a more precise recommendation connected with red and processed meat intake in the context of reproductive health.

\section{Conflict of Interest}

There is no conflict of interest. Project financially supported by Minister of Science and Higher Education in the range of the program entitled "Regional Initiative of Excellence" for the years 2019-2022, Project No. 010/RID/2018/19, amount of funding 12.000.000 PLN. 